\title{
Nota Científica \\ Potencialidades de Sclerocarya birrea em Chigubo província de Gaza, Moçambique
}

Jacob Miguel Bila ${ }^{1}$, Iva Carla Nunes de Carvalho Vaz

'Instituto de Investigação Agrária de Moçambique, Avenida das FPLM, 2698, CP 3658, Maputo, Moçambique

\author{
"Autor correspondente: \\ jacobila@yahoo.com.br \\ Termos para indexação: \\ Dendrometria \\ Amêndoa de canhu \\ Produto florestal não madeireiro
}

Index terms:

Forest mensuration

Canhu nut

Nonwood forest product

Histórico do artigo:

Recebido em 20/10/2015

Aprovado em 08/05/2017

Publicado em 30/06/2017

doi: 10.4336/2017.pfb.37.90.1059

\begin{abstract}
Resumo - Este trabalho teve como objetivo inventariar a ocorrência de Sclerocarya birrea (A.Rich) Hochst) no distrito de Chigubo, visando fornecer informação sobre seu potencial produtivo para exploração do óleo (canhu) pelas comunidades locais. Os dados de diâmetro à altura do peito (dap), altura total e comercial e os nomes das espécies foram levantados em dez conglomerados de quatro parcelas com 0,2 ha cada, estabelecidas aleatoriamente nas áreas, para determinar a estrutura horizontal da floresta, distribuição diamétrica e o volume de madeira por unidade da área. Posteriormente, foi determinada a quantidade de produção de amêndoa de canhu no distrito de Chigubo. Foi observada distribuição diamétrica do tipo "J" invertido, com cerca de 106 árvores ha-1 com dap $\geq 10 \mathrm{~cm}$, das quais $50,59 \%$ eram de $S$. birrea. A área basal total de espécies adultas foi de $34,26 \mathrm{~m}^{2} \mathrm{ha}^{-1}$, sendo $24,36 \mathrm{~m}^{2} \mathrm{ha}^{-1}(71,01 \%)$ de $S$. Birrea. A produção da amêndoa de $S$. birrea no distrito foi estimada em $13.345 \mathrm{~kg} \mathrm{ha}^{-1} \mathrm{ano}^{-1}$.
\end{abstract}

\section{Potential of Sclerocarya birrea in Chigubo, Gaza Province, Mozambique}

\begin{abstract}
The aim of this work was to assess the occurrence of Sclerocarya birrea in Chigubo district to provide information about its productive potential to promote the production of canhu oil by local communities. Diameter at breast height (DBH), total height and commercial height and species names were collected in 10 clusters of four plots with 0.2 ha each. The plots were randomly established in the area to determine horizontal forest structure, diameter distribution and timber volumes per unit area. Later on it was estimate de canhu nuts production in Chigubo district. We found diameter distribution in reverse J-shaped, 106 trees $^{-1} a^{-1}$, with $50.59 \%$ from $S$. birrea, and total basal area of adult species of $34.26 \mathrm{~m}^{2} \mathrm{ha}^{-1}$, with $24.36 \mathrm{~m}^{2} \mathrm{ha}^{-1}(71.01 \%)$ represented by $S$. birrea. Sclerocarya birrea nuts production was estimate as $13,345 \mathrm{~kg} \mathrm{ha}^{-1} \mathrm{year}^{-1}$.
\end{abstract}

A exploração de produtos florestais não madeireiros em Moçambique é praticada na sua maioria pelas comunidades locais para a satisfação das suas necessidades básicas (Forests..., 2014). Nestes termos, a sua quantificação é limitada ou quase inexistente. A espécie Sclerocarya birrea (A.Rich) Hochst, mais conhecida localmente como canhu, é uma dicoteledônea da família Anacardiaceae. É uma árvore de porte médio que pode atingir de 10 a $15 \mathrm{~m}$ de altura. Os frutos são carnudos quase esféricos, indescentes (fevereiro a junho) (Madumane, 2004). Tendo em conta as potencialidades do distrito na produção da amêndoa de canhu como matéria prima, o estabelecimento de uma fábrica de processamento de óleo de cozinha a partir de fruto de 
canhu seria de grande valia para as comunidades locais, pela oportunidade de alocação de mão-de-obra local. Portanto, a quantificação da produção de amêndoa de canhu no distrito é muito importante para a tomada de decisão sobre a implantação desta indústria.

Este trabalho tem como objetivo apresentar a estrutura horizontal da floresta, descrever a distribuição diamétrica e quantificar a produção anual do fruto de canhu no distrito de Chigubo, gerando informações que possam subsidiar o planejamento do seu manejo para produção de canhu pelas comunidades locais.

Esse trabalho foi desenvolvido no âmbito do Projeto "Fortalecimento das capacidades nacionais e das estruturas para a redução do risco de desastres e adaptação às mudanças climáticas", com financiamento do Programa das Nações Unidas para o Desenvolvimento (PNUD). Foi inicialmente realizado o inventário florestal da área, que é a base para a tomada de decisões em relação ao uso do solo.

O distrito de Chigubo fica situado a Norte da provincia de Gaza e está localizado entre os paralelos $33^{\circ} 30^{\prime} \mathrm{E}$ e $23^{\circ} 30^{\prime} \mathrm{S}$. Como limites, tem a Norte os distritos de Massangena e Chicualacuala; a Sul os distritos de Chibuto e Guijá; a Este a província de Inhambane (distritos de Funhalouro e Panda), e a Oeste os distritos de Chicualacuala e Mabalane (Utui et al., 2013).

Com uma superfície de $14.864 \mathrm{Km}^{2}$ e uma população recenseada em 1997 de 13.405 habitantes e estimada em 2005 em cerca de 16.698 habitantes, o distrito de Chigubo tem uma densidade populacional de $1,1 \mathrm{hab} \mathrm{km}^{-2}$. A população é jovem (47\% abaixo dos 15 anos de idade), majoritariamente feminina (taxa de masculinidade de $42 \%$ ) e de matriz totalmente rural (Utui et al., 2013).

O clima do distrito é do tipo árido, com uma precipitação média anual inferior a $500 \mathrm{~mm}$, e uma evapotranspiração potencial de referência (ETo) geralmente superior a $1.500 \mathrm{~mm}$. A maior parte da região apresenta temperaturas médias anuais superiores a $24^{\circ} \mathrm{C}$. A temperatura elevada agrava consideravelmente as condições de fraca precipitação, provocando deficiências de água superiores a $800 \mathrm{~mm}$ anuais (Moçambique, 2005).

Tais condições são agravadas pela grande irregularidade da quantidade de precipitação ao longo da estação chuvosa e por conseguinte a ocorrência de frequentes períodos secos durante o crescimento de culturas. A umidade relativa média anual é cerca de
$60 \%$ a $65 \%$ (Moçambique, 2005). O principal rio da região é o Changane, que banha a faixa leste do distrito, estabelecendo a fronteira com a província de Inhambane.

A maior parte da região tem altitudes inferiores a 200 m. Em geral, os solos são rasos, com zonas arenosas características da cobertura dos depósitos de Mananga. Destaca-se que os solos aluvionares que ocorrem ao longo da planície do rio Changane, embora com limitações decorrentes da presença de sais em excesso (i.e. sodicidade), são característicos de região seca e semiárida, com precipitações anuais baixas que variam de 200-400 mm (Moçambique, 2005).

As amostras foram constituídas por um conglomerado de quatro parcelas retangulares, com 0,2 ha cada ( $100 \mathrm{~m}$ x $20 \mathrm{~m}$ ). Estes conglomerados constituem um elemento estatístico básico (unidade de amostra). As parcelas estão localizadas a $300 \mathrm{~m}$ umas das outras a partir do inicio de cada lado de um quadrado de $400 \mathrm{~m}$ de lado.

Foi determinada a abundância, dominância e frequência absolutas e relativas. Foi também calculado o índice de valor de importância, pela média das abundâncias, dominâncias e frequências relativas e o volume individual e comercial por ha (Bila \& Mabjaia, 2012).

De acordo com Tomé et al. (2007), a distribuição de diâmetros de um povoamento corresponde à frequência das árvores de acordo com as classes de diâmetro previamente fixadas. A amplitude das classes de diâmetro pode ser ajustada em função da espécie e da dimensão das árvores, mas a amplitude mais usual é de $5 \mathrm{~cm}$.

A produção anual de canhu por árvore é de sete mil frutos por ano, que corresponde a $500 \mathrm{~kg}$ árvore ${ }^{-1} \mathrm{ano}^{-1}$ (Directorate Plant Production, 2010). Conhecendo o número de árvores encontradas na avaliação quantitativa, estimou-se a quantidade de frutos produzidos no distrito de Chigubo.

Nas áreas florestais do distrito de Chigubo foram encontradas cerca de 106 árvores ha ${ }^{-1}$ com diâmetro a $1,30 \mathrm{~m}$ do solo (DAP) $\geq 10 \mathrm{~cm}$, das quais $50,59 \%$ eram de Sclerocarya birrea. A área basal total de espécies adultas é de $34,26 \mathrm{~m}^{2}$ ha-1, dos quais $24,36 \mathrm{~m}^{2}$ ha ${ }^{-1}$ são de $S$. birrea o que corresponde a $71,0 \%$. A frequência absoluta de S. birrea foi de $100 \%$, tendo em conta que esta espécie foi observada em todas as parcelas amostrais, seguida das espécies Acacia nigrescensis, Albizia forbesii, Euphorbia quadrangularise e Spirostachys africana, com $40 \%$ de frequência absoluta (Tabela 1). 
Tabela 1. Abundância, dominância e frequência das espécies encontradas em floresta natural, em Chigubo.

\begin{tabular}{|c|c|c|c|c|c|c|c|c|}
\hline \multirow[b]{2}{*}{ Espécie } & \multirow[b]{2}{*}{ Nome comum } & \multicolumn{2}{|c|}{ Abundância } & \multicolumn{2}{|c|}{ Dominância } & \multicolumn{3}{|c|}{ Frequência } \\
\hline & & $\begin{array}{l}\text { Absoluta } \\
\left(\mathbf{N}^{0} \text { árv ha }\right. \\
\text { he })\end{array}$ & $\begin{array}{c}\text { Relativa } \\
(\%)\end{array}$ & $\begin{array}{l}\text { Absoluta } \\
\left(\mathbf{m}^{2} \mathbf{h a}^{-1}\right)\end{array}$ & $\begin{array}{c}\text { Relativa } \\
(\%)\end{array}$ & $\begin{array}{c}\text { Absoluta } \\
\text { (Parcelas) }\end{array}$ & $\begin{array}{c}\text { Relativa } \\
\text { (\%) }\end{array}$ & IVI \\
\hline Abrus precatorius & Sesane & 0,25 & 0,24 & 0,08 & 0,24 & 1 & 1,3 & 1,78 \\
\hline Acacia nigrescensis & Incaia & 4,00 & 3,79 & 0,61 & 1,78 & 2 & 2,6 & 8,17 \\
\hline Acacia nilotica & Nicaia & 11,63 & 11,02 & 2,42 & 7,07 & 4 & 5,19 & 23,29 \\
\hline Acacia sp. & Caia & 1,13 & 1,07 & 0,24 & 0,70 & 1 & 1,3 & 3,06 \\
\hline Afzelia quanzensis & Chanfuta & 0,25 & 0,24 & 0,15 & 0,42 & 2 & 2,6 & 3,26 \\
\hline Albizia anthelminthica & Dzangalagwva & 0,5 & 0,47 & 0,05 & 0,13 & 1 & 1,3 & 1,9 \\
\hline Albizzia anthelmintica & Dzigulagumva & 0,38 & 0,36 & 0,03 & 0,07 & 3 & 3,9 & 4,33 \\
\hline Albizzia brevifolia & Luhani & 0,25 & 0,24 & 0,06 & 0,17 & 1 & 1,3 & 1,7 \\
\hline Albizzia forbesii & Nala & 3,38 & 3,2 & 0,33 & 0,97 & 4 & 5,19 & 9,36 \\
\hline Androstachys johnsonii & Cimbirre & 0,25 & 0,24 & 0,03 & 0,10 & 1 & 1,3 & 1,64 \\
\hline Brachystegia spiciformis & Panguii & 0,13 & 0,12 & 0,01 & 0,02 & 1 & 1,3 & 1,44 \\
\hline Brachystegia utilis & Caunza & 0,25 & 0,24 & 0,32 & 0,93 & 1 & 1,3 & 2,46 \\
\hline Bridelia cathartica & Tlatlangate & 0,63 & 0,59 & 0,06 & 0,17 & 2 & 2,6 & 3,36 \\
\hline Cassia abbreviate & Lumanhana & 0,25 & 0,24 & 0,02 & 0,06 & 1 & 1,3 & 1,59 \\
\hline Cassia abbreviate & Lumanhana & 0,25 & 0,24 & 0,02 & 0,06 & 1 & 1,3 & 1,59 \\
\hline Colophospermum mopane & Mopani & 1,63 & 1,54 & 0,45 & 1,32 & 1 & 1,3 & 4,16 \\
\hline Combretum apiculatum & Xivondjoane & 0,38 & 0,36 & 0,03 & 0,09 & 2 & 2,6 & 3,05 \\
\hline Combretum imberbe & Xihondzuane & 0,63 & 0,59 & 0,05 & 0,14 & 1 & 1,3 & 2,03 \\
\hline Combretum molle & Chucutse & 0,38 & 0,36 & 0,14 & 0,42 & 1 & 1,3 & 2,08 \\
\hline Dalbergia obovata & Landulu & 0,13 & 0,12 & 0,01 & 0,04 & 1 & 1,3 & 1,45 \\
\hline Diospyros sp. & Xacuari & 0,5 & 0,47 & 0,06 & 0,19 & 2 & 2,6 & 3,26 \\
\hline Dolichandrone ailba & Tsani & 0,13 & 0,12 & 0,04 & 0,11 & 1 & 1,3 & 1,53 \\
\hline Euphorbia quadrangularis & Tsotso & 6,75 & 6,4 & 1,09 & 3,19 & 4 & 5,19 & 14,79 \\
\hline Garcinia huillensis & Tchisso & 0,75 & 0,71 & 0,11 & 0,33 & 1 & 1,3 & 2,34 \\
\hline Guibourtia coleosperma & Xacatipreto & 1,38 & 1,3 & 0,27 & 0,80 & 1 & 1,3 & 3,4 \\
\hline Kigelia africana & Mpfungula & 0,13 & 0,12 & 0,01 & 0,03 & 1 & 1,3 & 1,44 \\
\hline Lannea stuhlmannii & Danicomo & 1,75 & 1,66 & 0,32 & 0,95 & 2 & 2,6 & 5,2 \\
\hline Lonchocarpus capassa & Mubandza & 0,75 & 0,71 & 0,20 & 0,58 & 1 & 1,3 & 2,59 \\
\hline Maerua juncea & Xipinga & 0,38 & 0,36 & 0,03 & 0,09 & 1 & 1,3 & 1,74 \\
\hline Manilkara discolor & Nwambo & 1,13 & 1,07 & 0,08 & 0,24 & 1 & 1,3 & 2,61 \\
\hline Melanodiscus oblongus & Xacuari & 0,88 & 0,83 & 0,25 & 0,74 & 1 & 1,3 & 2,87 \\
\hline Olax dissitiflora & Lijamuntani & 1,00 & 0,95 & 0,39 & 1,15 & 1 & 1,3 & 3,4 \\
\hline Ozoroa obovata & Chinungo & 1,88 & 1,78 & 0,44 & 1,27 & 1 & 1,3 & 4,35 \\
\hline Ozoroa obovata & Catassaro & 0,25 & 0,24 & 0,18 & 0,52 & 1 & 1,3 & 2,05 \\
\hline Pterocarpus rotundifolius & Tsandzanhompfu & 0,13 & 0,12 & 0,02 & 0,04 & 1 & 1,3 & 1,46 \\
\hline Sarcostemma viminale & Xiguetane & 0,25 & 0,24 & 0,05 & 0,15 & 1 & 1,3 & 1,69 \\
\hline Sclerocaryabirrea & Canhoeiro & 53,38 & 50,59 & 24,33 & 71,01 & 10 & 12,99 & 134,59 \\
\hline Spirostachys africana & Sandalo & 2,13 & 2,01 & 0,38 & 1,1 & 4 & 5,19 & 8,31 \\
\hline
\end{tabular}

Continua... 
Tabela 1. Continuação.

\begin{tabular}{|c|c|c|c|c|c|c|c|c|}
\hline \multirow[b]{2}{*}{ Espécie } & \multirow[b]{2}{*}{ Nome comum } & \multicolumn{2}{|c|}{ Abundância } & \multicolumn{2}{|c|}{ Dominância } & \multicolumn{3}{|c|}{ Frequência } \\
\hline & & $\begin{array}{c}\text { Absoluta } \\
\left(\mathbf{N}^{0} \text { árv ha' }\right.\end{array}$ & $\begin{array}{c}\text { Relativa } \\
(\%)\end{array}$ & $\begin{array}{c}\text { Absoluta } \\
\left(\mathbf{m}^{2} \mathbf{h a}^{-1}\right)\end{array}$ & $\begin{array}{c}\text { Relativa } \\
(\%)\end{array}$ & $\begin{array}{l}\text { Absoluta } \\
\text { (Parcelas) }\end{array}$ & $\begin{array}{c}\text { Relativa } \\
(\%)\end{array}$ & IVI \\
\hline Sterculia rogersii & Xilusuane & 0,5 & 0,47 & 0,07 & 0,19 & 3 & 3,9 & 4,56 \\
\hline Sterculia schliebenii & Semanhe & 1,13 & 1,07 & 0,18 & 0,53 & 1 & 1,3 & 2,9 \\
\hline Strychnos madagascariensis & Macuacua & 2,25 & 2,13 & 0,26 & 0,75 & 2 & 2,6 & 5,48 \\
\hline Terminalia sericea & Conola & 0,13 & 0,12 & 0,01 & 0,02 & 1 & 1,3 & 1,44 \\
\hline Terminalia sericea & Xane & 0,13 & 0,12 & 0,03 & 0,09 & 1 & 1,3 & 1,51 \\
\hline Tricholia emetic & Mafurra & 0,13 & 0,12 & 0,02 & 0,05 & 1 & 1,3 & 1,47 \\
\hline Xanthocercis zambesiaca & Lharu & 1,13 & 1,07 & 0,33 & 0,96 & 1 & 1,3 & 3,33 \\
\hline Total & & 105,5 & 100 & 34,26 & 100 & 10 & 100 & 300 \\
\hline
\end{tabular}

A distribuição diamétrica de todas as espécies apresenta uma curva de distribuição de tipo J-invertido (Figura 1), sendo que 60\% dos indivíduos estão concentrados nas duas primeiras classes diamétricas (de $10 \mathrm{~cm}$ a $20 \mathrm{~cm}$ ). Este tipo de distribuição mostra que existe um balanço entre o recrutamento e a mortalidade, o que garante a sustentabilidade da floresta. Resultados semelhantes foram observados por Geldenhuys (1996) e Ribeiro et al. (2002).

Analisando-se a distribuição diamétrica de S. birrea (Figura 2), nota-se também uma tendência da distribuição do tipo J-invertido, onde as classes diamétricas mais jovens são abundantemente representadas, o que significa que o potencial de reposição da floresta após a morte (natural ou não) dos indivíduos adultos é alto (Ribeiro et al., 2002),

A abundância de $S$. birrea é de 53.38 árvores ha-1, tendo-se estimado a produção média do fruto de canhu em $26.690 \mathrm{~kg} \mathrm{ha}^{-1}$ ano $^{-1}$. A relação peso do fruto e peso de amêndoa é de 2:1 (Madumane, 2004). Portanto, a produção anual da amêndoa por árvore é de $250 \mathrm{~kg} \mathrm{arv}^{-1}$ ano $^{-1}$, correspondendo a uma produção de $13.345 \mathrm{~kg} \mathrm{ano}^{-1} \mathrm{ha}^{-1}$.

Considerando a área do distrito de $14.864 \mathrm{~km}^{2}$ o que corresponde a 1.486,4 ha, a produção anual da amêndoa de canhu ao nível de distrito é de cerca de 19.836 ton. Essa produção seria suficiente para o abastecimento da fábrica.

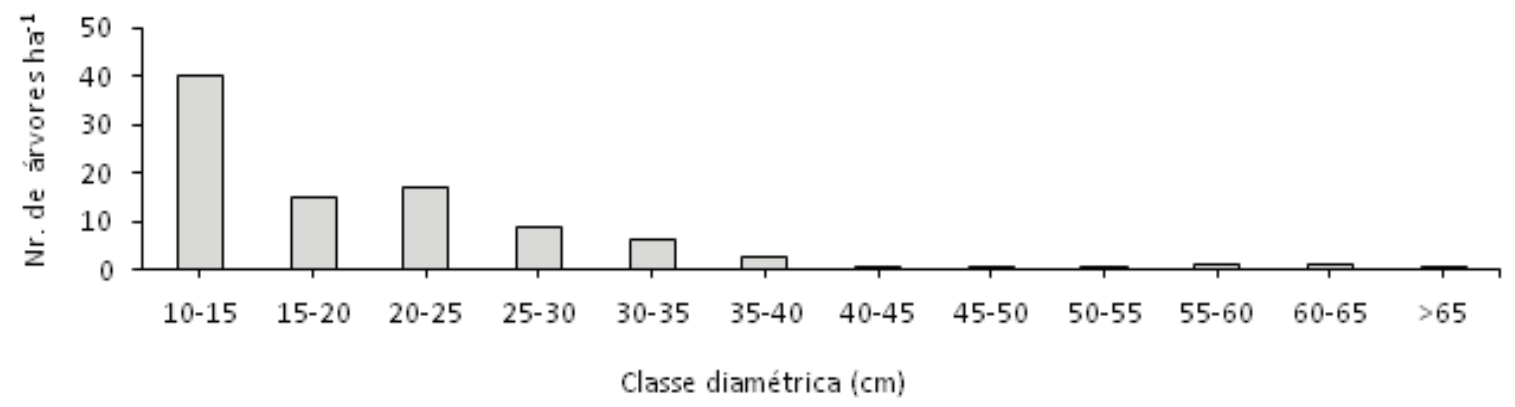

Figura 1. Distribuição diamétrica de todas as espécies, em floresta natural no Distrito de Chigubo. 


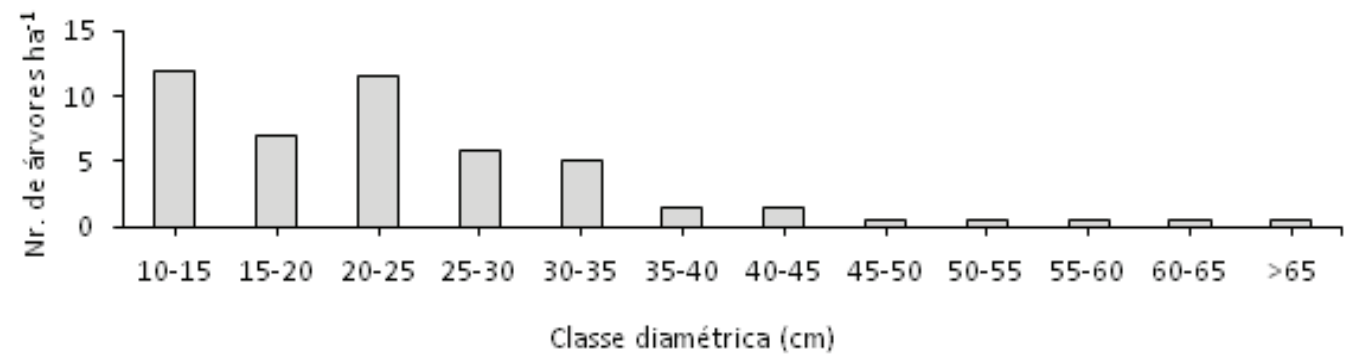

Figura 2. Distribuição diamétrica de Sclerocarya birrea que ocorre em Chigubo.

\section{Conclusões}

A espécie Sclerocarya birrea ocorre em Chigubo com uma abundância de 53.38 árvores ha ${ }^{-1}$, o que corresponde a $50,59 \%$ da abundância total de todas as espécies. A distribuição diamétrica das espécies mostra que ainda existe um potencial de crescimento na área, pelo manejo da regeneração contínua da espécie. A produção da amêndoa de canhu no distrito foi estimada em cerca de $13.345 \mathrm{~kg}$ ano $^{-1} \mathrm{ha}^{-1}$, que provavelmente suficiente para o abastecimento da fábrica de processamento de óleo vegetal.

\section{Referências}

Bila, J M. \& Mabjaia, N. Crescimento e fitossociologia de uma floresta com Colophospermum mopane, em Mabalane, Província de Gaza, Moçambique. Pesquisa Florestal Brasileira, v. 32, n. 72, p. 421-427, 2012. DOI: 10.4336/2012.pfb.32.72.421.

Directorate Plant Production. Marula: production guideline. Pretoria: Department of Agriculture, Forest and Fisheries, 2010. 20 p.

Forests in Mozambique face extinction. WRM Monthly Bulletin,, n. 205, 2014. Avaliable from: <http://wrm.org.uy/bulletins/issue-205/>. Access on: 09 may 2017.
Geldenhuys, C. J. Options for sustainable harvesting of timber products from Woodlands: example from Southern Africa. In: Sustainable management of indigenous forests in the dry tropics: proceedings of an international conference. Kadoma, Zimbabwe, 1996. Zimbabwe: Forestry Commission; [Stockholm]: SAREC: SIDA, 1996.

Madumane, J. G. F. Factores que influenciam a regeneração e estabelecimento natural de Screrocarya birrea-Kanhe (A. Rich) Hochst (1844). 2004. 54 f. Tese (Licenciatura em Engenharia Florestal) - Faculdade de Agronomia e Engenharia Florestal, Maputo.

Moçambique. Ministério de Administração Estatal. Direção Nacional da Administração Local. Província de Gaza. Perfil do Distrito de Chigubo. 2005. Avaliable from: <www.portaldogoverno.gov.mz/ por/content/download/2959/23847/.../Chigubo.pdf> Access on: 12 May 2017.

Ribeiro, N. et al. Caracterização ecológica da floresta de galeria do Rio Mecubúri na reserva florestal de Mecuburi, Província de Nampula. Maputo: UEM-DNFFB/CEF, 2002. 37 p.

Tomé, M. et al. Relações hipsométricas geral para Eucalyptus globulus Labill em Protugal, Silva Lusitana, v. 15, n. 1, p. 55, 2007.

Utui, J. et al. Estatística do Distrito de Chigubo. Maputo: Instituto Nacional de Estatística, 2013. 32 p. 
